\title{
A multi-criteria model analysis framework for assessing integrated water-energy system transformation pathways
}

Article · January 2017

DOI: 10.1016/j.apenergy.2016.12.142

CITATION

1

6 authors, including:
READS

105

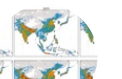

(2)

\section{Simon Parkinson}

University of Victoria / International Institute fo.

17 PUBLICATIONS 294 CITATIONS

SEE PROFILE

\section{Ned Djilali}

University of Victoria

267 PUBLICATIONS $\quad 6,950$ CITATIONS

SEE PROFILE

Some of the authors of this publication are also working on these related projects: 


\title{
in print, Applied Energy 2017 \\ DOI: 10.1016/j.apenergy.2016.12.142
}

\section{A multi-criteria infrastructure planning framework for integrated water-energy systems}

\author{
Simon C. Parkinson ${ }^{\mathrm{a}, \mathrm{b}, *}$, Marek Makowski ${ }^{\mathrm{b}, \mathrm{c}, * *}$, Volker Krey $^{\mathrm{b}}$, Khaled Sedraoui ${ }^{\mathrm{d}}$, Abdulrahman .H. Almasoud ${ }^{\mathrm{d}}$, Ned \\ Djilali $^{\mathrm{a}, \mathrm{d}}$ \\ ${ }^{a}$ Institute for Integrated Energy Systems, University of Victoria, Canada \\ ${ }^{b}$ Energy Program, International Institute for Applied Systems Analysis, Austria \\ ${ }^{c}$ Systems Research Institute, Polish Academy of Sciences, Poland \\ ${ }^{d}$ Renewable Energy Research Group, King Abdulaziz University, Saudi Arabia
}

\begin{abstract}
Sustainable development objectives surrounding water and energy systems are increasingly interdependent, and yet the associated performance metrics are often distinct. Regional planners tasked with designing future supply systems therefore require multi-criteria analysis methods and tools to determine a suitable combination of technologies and scale of investments. Previous research focused on optimizing system development strategy with respect to a single design objective, leading to potentially negative outcomes for other important sustainability metrics. This paper addresses this limitation, and presents a flexible and interactive multi-criteria model analysis framework and its application to long-term energy and freshwater supply planning at national or regional scales. The framework incorporates a linear systems-engineering model of the coupled supply technologies and inter-provincial electricity and water transmission networks. The multi-criteria analysis approach enables the interactive specification of diverse decision-making preferences for disparate criteria, and leads to learning on trade-offs between the resulting criteria values of the corresponding Pareto-optimal solutions. A case study of the water-stressed nation of Saudi Arabia explores preferences combining aspiration and reservation levels in terms of cost, water sustainability and $\mathrm{CO}_{2}$ emissions. The analysis reveals a suite of trade-off solutions, in which potential integrated system configurations remain relatively ambitious from both an economic and environmental perspective. The identified cost savings would have a major impact on the affordability of water and electricity services in Saudi Arabia.
\end{abstract}

Keywords:

Water-energy nexus; climate change mitigation; energy systems analysis; capacity expansion planning;

Pareto-optimal solutions; Saudi Arabia

\footnotetext{
*Corresponding author. Email address: scp@uvic.ca

**Corresponding author. Email address: marek@iiasa.ac.at
} 


\section{Introduction}

Water plays a key role in the supply of energy in many regions globally, primarily for thermal power plant cooling and hydropower generation [1]. Constraints on the availability of water resources in these regions therefore pose risks to energy service reliability. At the same time, a significant amount of energy is required to extract, treat and distribute freshwater resources [2]. Constraints on the supply of freshwater services therefore pose risks of additional energy requirements. Moreover, energy and freshwater are required for meeting the development goals of societies. These interdependencies are often referred to as the water-energy nexus, and promote integrated planning of water and energy infrastructure systems.

Infrastructure here refers to the technologies or processes that enable supply of energy and water services to consumers. Planners tasked with designing regional energy and freshwater infrastructures are faced with a plethora of technologies and a wide variety of economic, social and environmental conditions, which make it difficult to decide which technologies to invest in and promote, and in what order. The optimal combination of technologies and level of investments will be difficult to determine without appropriate analysis methods and tools. From this perspective, mathematical programming models have provided critical decision support by enabling planners to identify system designs that perform well under anticipated operational conditions [3-8].

Previous studies explored impacts of water constraints on energy system operation by coupling water supply and electricity generation dispatch models [9-13]. Several other previous studies note the importance of future capacity decisions (the size and location of technologies) in terms of enabling effective adaptation to future water constraints, and examined the impact of water availability on the development of regional power systems by adding explicit water constraints to an optimal infrastructure planning model [14-21]. Water constraints are found to primarily cause a shift towards water-efficient cooling technology for thermal power generation, as well as increased siting in regions with greater access to water availability [18]. Increased hydrologic variability under climate change was also found to cause further long-term capacity challenges in regions where hydropower plays an important role in electricity supply $[15,20]$. A key limitation of these previous analyses of water constraints is the inability to incorporate feedbacks from future water supply development, which will impact the availability of water for energy and water-related energy demand. To reconcile development interdependencies, a number of other studies link freshwater and energy infrastructure planning models directly [22-29]. This approach enables modeling of system configurations that adapt to undesirable interactions between water and energy during infrastructure development.

Most previous coupled planning models focus on identifying system configurations that minimize costs or maximize consumer surplus. Yet, there are often other social or environmental objectives of concern to regional decisionmakers and stakeholders, thus requiring a more integrated approach to assessing system performance [30]. Metrics of interest include limiting greenhouse gas emissions and air pollution, and securing food, water and energy resources. Previous analyses addressed such objectives as constraints, values of which were explored using parametric optimization [16, 27, 28, 31]. Parametrization of constraints requires not only skilled analysts but also specification of a large 
number of optimization problems, many of which are either infeasible or result in dominated (inefficient) solutions. Multi-criteria analysis (MCA) of discrete alternatives can be applied to the results of parametric model optimization [31], but such a two-stage process is by far less effective than a direct linking of the model with the MCA tool. Another popular approach is based on weighted-sum criteria aggregation into a composite goal function. This approach has, however, serious shortcomings [32], e.g.,: (1) in some situations the same solution is returned even if substantial changes are made to the weights; (2) many efficient solutions ${ }^{1}$ cannot be obtained by varying the weights; and (3) increasing a weight does not guarantee improvement of the corresponding criterion value.

In this context, formal MCA methods offer an improvement to traditional optimization approaches, as illustrated by a sample of applications relevant to the case study presented in this paper [33-36]. MCA supports analysis of tradeoffs between all relevant objectives, and interactive exploration of diverse efficient solutions across multiple objectives. Despite the potential to apply this type of methodology and tools to effectively model coupled economicenvironmental decision-making [37], application of MCA to the integrated planning of energy and water systems has been limited to cooling technology choices in the power sector [38].

This paper presents a novel systems analysis tool for integrated regional planning of energy and freshwater supply systems. The framework incorporates a multi-objective decision support system to enable analysis of long-term infrastructure strategies that balance economic, energy and water sustainability objectives. The integrated decision support framework is demonstrated within a case study of the water-stressed, carbon-intensive nation of Saudi Arabia The results of the analysis provide important new insights into the following research questions:

- How can multiple design criteria be incorporated into long-term infrastructure planning models covering both the water and energy supply sectors?

- What is the potential scale of tradeoffs between environmental and economic development objectives in the case study region, and how might relaxing ambition levels for water and energy sustainability impact affordability?

The paper proceeds as follows. The methodology of model-based decision-support and its implementation for integrated water-energy systems is presented in Section 2 . The case study demonstrating model application is described in Section 3 followed by the discussion of results in Section 4. Conclusions from the research are summarized in Section 5.

\section{Methodology}

This section presents the approach for coupled water-energy supply planning and its integration with the MCA methods and tools. The framework is based around a water-energy infrastructure planning model developed previously for Saudi Arabia [28]. Previous research with this framework demonstrated that transitioning away from nonrenewable

${ }^{1}$ Solutions are called efficient or Pareto-optimal if there exists no other solution for which at least one criterion can be made better without sacrificing performance of the criteria. 
groundwater use by the year 2050 in Saudi Arabia could increase electricity demand by more than $40 \%$ relative to 2010, due to rapid development of desalination and water conveyance infrastructure, and require investments similar to strategies aimed at transitioning away from fossil fuels in the electricity sector. These results highlight the need to incorporate multiple policy objectives into system design, and is the key feature of the enhanced MCA tool proposed in the current study. Following a description of the mathematical model for coupled water-energy supply planning, we discuss its integration with the applied MCA methodology. Finally, we describe the input data and scenarios explored in the case study demonstrating model application.

\subsection{A core model for integrated water-energy infrastructure development}

The planning challenge dealt with in this paper is the sustainable long-term development of water and energy systems. These decisions are typically made at national or regional-scales, and encompass choices surrounding the capacity of existing and future infrastructure. Capacity decisions are key design parameters for energy and water supply planners due to the relationship with geographical constraints, investment costs and long-term structural inertia of the supply systems [4]. Capacity choices incorporate both the size and location of new technologies, as well as the operational management (activity) of the technologies over the planning horizon. Strategizing capacity decisions is also commonly referred to as capacity expansion planning, but may also entail reductions in system capacity in situations where reduced demands are projected. Due to the impact on long-term structural inertia, capacity decisions are usually assessed over multi-decadal time periods. Performance criteria of primary concern include service reliability, end-use prices and environmental impacts.

Water and energy resource potentials represent an important input to any capacity planning approach, and vary significantly across resources, time and geographic location. Transporting water and energy from one location to another also requires massive investment in network infrastructure, with long-distance water conveyance presenting further interdependencies due to the energy required for pumping. Planning models incorporating spatially resolved infrastructure systems will be needed to understand the implications of local constraints and transmission for longterm development strategy [24, 39-42]. Yet, there is also a need to maintain an adequate temporal resolution in order to capture operational constraints occurring primarily in the electricity sector [43]. Moreover, spatial units typical in water resource management are geophysically-based and do not necessarily align with administrative units typical in energy supply planning (e.g., national, provincial, utility, etc.). The spatial mismatch may require disaggregation of spatial decision-making units in order to converge on a common resolution across energy and water systems [44]. The added complexity will be additionally demanding to accommodate in mathematical models containing an already diverse range of technologies and processes. Maintaining a careful balance between spatial and temporal scales when developing integrated water-energy models for long-term planning purposes is thus a critical challenge for regional planners, and scoping will depend on the specific research question (e.g., transmission expansion, emissions mitigation, groundwater depletion, etc.) and characteristics of the study region (interconnectivity of basins/aquifers, population density, income-level, etc.). 
In this paper, we adapt the Saudi Arabia Electricity-Water Planning model (SEWP): an integrated supply planning framework that incorporates simulataneous capacity decisions in the water and electric power sectors. The framework includes a diverse range of technologies including most power generation types (e.g., natural gas combined-cycle, concentrating solar power, etc.) and water supply technologies (e.g., groundwater extraction, desalination, wastewater recycling, etc.). Thermal power plants are further distinguished by cooling technology (e.g., once-through, recirculating, etc.). The study region is broken into the 13 provincial administrative regions, with expandable electricity and freshwater transmission between provinces included in the capacity planning decisions. To explore impacts of national policy and path-dependency on technology deployment, SEWP focuses on a planning horizon of 2010 to 2050 in 5-year segments, with each time-step solved concurrently. Each modeled year is broken into monthly timeslices to enable treatment of seasonal effects, such as the potential mismatch between available supply and demand. For computational efficiency, the current version of SEWP considers linear relantionships between variables. Although designed specifically for application to infrastructure planning in Saudi Arabia, the approach is readily adaptable to other regional situations.

SEWP ensures a physical representation of resource conversion across a set of $R$ resources, $I$ spatially distributed regions, and $T$ temporally distributed decision making intervals. For each resource $r \in R$, location $i \in I$ and time-step $t \in T$, the managed supply must exceed the exogenous demand:

$$
Q(r, i, t)+\Delta S(r, i, t) \geq D(r, i, t)
$$

where $Q$ is the managed flow from supply technologies, $\Delta S$ is the managed flow from storage, and $D$ is the exogenous demand. The managed flow from supply technologies includes consumption and production of different energy and water resources at the technology-level, and can be modeled consistently using appropriate functional relationships that link technology activity to net resource availability. SEWP considers a diverse set of $P$ technologies capable of operating in a set of $O$ operational modes, and calculates the managed flow of resource $r \in R$ from a specific technology $p \in P$ using input activity ratios $\epsilon^{i n}$ and output activity ratios $\epsilon^{\text {out }}$. The activity ratios represent the average rate at which a certain technology consumes or produces a certain resource per unit of activity-level. Operational modes are distinguished to enable representation of diverse operating costs and efficiencies for a single technology type. To allow for spatial transfers of water and electricity via conveyance or transmission infrastructure, net resource flows in each region $i \in I$ incorporates inputs produced and consumed in that region, as well as from other regions $j \in I$. Summing across regions, modes and technologies yields the managed flow for each resource in each region and time step:

$$
Q(r, i, t)=\sum_{p, o, j}\left[\epsilon^{o u t}(r, p, o, j, i, t) \cdot x(p, o, j, t)-\epsilon^{i n}(r, p, o, i, j, t) \cdot x(p, o, i, t)\right]
$$

where $x$ is the activity-level of a specific technology. The change in storage-level is equivalent to the difference 
between the levels across decision-making intervals:

$$
\Delta S(r, i, t)=s(r, i, t)-s(r, i, t+1)
$$

where $s$ is the storage-level. Surface water reservoirs and potable storage at end-use are the only between-month storage technologies currently included in SEWP. Level-dependent losses are important for surface water reservoirs (evaporation is proportional to surface area), and can be accounted for using linearized area-volume relationships [45]. Saudi Arabia contains relatively little exploitable surface water and associated storage, and for this reason, volume-dependent losses are neglected. Due to uncertainties surrounding the scale of the resource and complexities of hydro-geological modeling, groundwater storage is incorporated into SEWP as a model criteria (section 2.2).

The activity-level of each technology is constrained in SEWP by the available capacity:

$$
\phi(p, i, t) \cdot z(p, i, t)-\sum_{o} \sigma(p, o, i, t) \cdot x(p, o, i, t) \geq 0
$$

where $z$ is the installed capacity, $\phi$ is the fraction of installed capacity that is available (or the capacity factor), and $\sigma$ is the rate at which a particular operational mode utilizes capacity. Certain operational modes are allowed to consume more capacity than others in the model to reflect e.g., capacity impacts of scheduling flexible reserve generation in the electricity sector [46]. SEWP includes incremental capacity expansion decisions $u$ that alleviate capacity constraints. Incremental capacity retirements $w$ are also modeled as decision variables to allow representation of finite technology lifecycles. The installed capacity of a particular technology is given by:

$$
z(p, i, t)-z(p, i, t+1)+u(p, i, t)-w(p, i, t)=0
$$

Likewise, storage capacity $c$ constrains storage levels, incremental new storage capacity $b$ can be used to alleviate constraints on storage levels, and incremental storage retirements $d$ reduce installed storage capacity:

$$
\psi(r, i, t) \cdot c(r, i, t)-s(r, i, t) \geq 0
$$

$$
c(r, i, t)-c(r, i, t+1)+b(r, i, t)-d(r, i, t)=0
$$

where $\psi$ is the fraction of installed storage capacity that is active. In the case reported in this paper, capacities are modeled by continuous variables. The authors are aware that integer variables enable modeling the effects of reduced unit costs with increasing unit size (i.e., economies-of-scale), which provides insight into the benefits of distributed or centralized supply configurations $[26,40]$. However, the choice of continuous variables is justified by two arguments. First, the obtained capacity values usually provide a good approximation. Second, and most importantly, due to the model size its mixed-integer formulation would require qualitatively more computational resources. 
Upper and lower bounds are further imposed on the capacity and activity variables to reflect e.g., resource availability, excess supply and existing infrastructure. Other additional contraints address operational policies such as technology retirements, inter-annual reservoir sustainability and electricity system flexibility. A detailed account of these relationships can be found elsewhere [28], and for brevity are not repeated here.

\subsection{Multi-criteria model analysis}

A vector of outcome variables $\mathbf{y}$ can be used for measuring various consequences of the simulated development strategy in SEWP. Outcome variables are often named differently (e.g. criteria, objectives, goals, metrics, performance indices, etc.). A vector of algebraic relations $\mathbf{F}$ are defined that convert decisions variables to outcomes:

$$
\mathbf{y}=\mathbf{F}(\mathbf{v}), \mathbf{v} \in V_{o}
$$

where $\mathbf{v}$ is the vector of model decision-variables (the activity and capacity of the technologies introduced in the previous section), and $V_{0}$ is the set of feasible solutions (admissible due to the physical and logical constraints introduced in the previous section).

Past application of SEWP focused on a single objective: minimize total discounted system costs over the planning horizon. This formulation requires a unique specification of a goal function that adequately represents system cost. Capital and operational cost parameters for each technology are input to SEWP and multplied by the corresponding capacity or activity variable to estimate the cost contribution. Discounting is then used to translate future costs into an estimated present value. In the single-objective formulation, preferences for outcomes, including available budget, requires a re-definition of the set of feasible solutions $V_{0}$ by $V_{1}: V_{1}=V_{0} \cap \mathbf{P}$, where $\mathbf{P}$ is the set of outcomes conforming to the decision-making preferences. In some cases the preferences are too ambitious, e.g., tight constraints on the budget actually shrinks the set of feasible solutions to a small subset (which ignores many possibly interesting solutions), or even results in an empty set $V_{1}$, which in turn makes the underlying optimization problem infeasible.

Alternatively, preferences for multiple objectives might be obtained based on linear weighted-sum criteria aggregation into a composite goal function. This approach has the serious shortcomings mentioned in the introductory section [32]. In this paper, an achievement scalarizing function (ASF) serves as the goal function in the mathematical programming analysis built on the core model described in the previous section. The ASF is defined through criteria achievement functions (CAFs) specified for each objective independently. The role of the CAFs is to provide a common measure for criteria performance, typically defined in different metrics and scales. We utilize a modified version of the reference point methodology [37, 47], where each CAF is parametrized by two values specified by the user, namely aspiration and reservation levels, which correspond to the criterion values that are desired and worst acceptable, respectively. In this context, a CAF for the $k$-th criterion is denoted by:

$$
u_{k}=f_{k}\left(q_{k}, \bar{q}_{k}, \underline{q}_{k}\right),
$$


where $f_{k}(\cdot)$ is a strictly monotone concave function (decreasing for minimized, and increasing for maximized criteria, respectively), and $q_{k}, \bar{q}_{k}, \underline{q}_{k}$ are the criterion value, aspiration, and reservation levels, respectively. Values of $q_{k}$ are defined by the corresponding outcome variables of the analyzed core model (i.e., $q_{k}=y_{k}$ ). The $f_{k}(\cdot)$ are usually defined as piece-wise linear functions with linear segments determined by the utopia, aspiration, reservation, and nadir values [48]. The utopia point $\mathbf{U}$ is defined by a vector composed of the best values of all considered criteria. Utopia components are easily computed through the so-called selfish optimizations (i.e., optimizing each criterion separately). The nadir point $\mathbf{N}$ is defined by the worst values of the criteria within the Pareto-set. The piece-wise linear functions represent the human values related to satisfaction and regret, and also have a nice mathematical property; namely, the underlying multi-criteria optimization model remains linear for linear core models. A correctly implemented multicriteria model analysis framework does not impose any restrictions on the feasibility of the aspiration and reservation values, other than two exceptions: (1) the reservation is lower/higher than aspiration for minimized/maximized criterion, respectively; and (2) the aspiration and reservation values are between the corresponding utopia and nadir values.

The CAF values have a very easy and intuitive interpretation in terms of the degree of satisfaction from the corresponding value of the criterion. Values of 1 and 0 indicate that the value of the criterion exactly meets the aspiration and reservation values, respectively. CAF values between 0 and 1 can be interpreted as the degree of satisfaction of the criterion value, i.e., to what extent this value is close to the aspiration level and far away from the reservation level. These interpretations correspond to the interpretation of the membership function from fuzzy set theory [48]. In fact, the CAF extends the membership function concept because the CAF also takes negative values (for criteria values worse than the reservation), and values greater than one (for criteria values better than the aspiration). This extension is necessary for proper handling of any $\bar{q}_{k}$ and $\underline{q}_{k}$, which in turn frees the users from concerns regarding attainability of the considered aspiration and reservation levels.

The ASF is defined by:

$$
\mathcal{S}=\min _{k \in K_{a}}\left(u_{k}\right)+\frac{\epsilon}{K} \cdot \sum_{k=1}^{K} u_{k}
$$

where $K_{a}$ is the subset of active criteria, $u_{k}$ are defined by (9), and $\epsilon$ is a small positive number. The first term causes improvement of the worst performing (in terms of the corresponding CAF) criterion. The second term assures that the optimal solution provided for maximization of the ASF is indeed Pareto-optimal [37, 49]. Maximization of (10) for $\mathbf{v} \in V_{o}$ generates a properly efficient solution aligned with the user's criteria preferences.

Implementation of the MCA methods described in this paper is accomplished with the Integrated Modeling Environment Project's online Multiple Criteria Model Analysis (MCMA) framework [50]. The approach is outlined in Appendix A. 


\section{Case study}

The focus of the Saudi Arabia case study analysis are infrastructure strategies that are efficient at simultaneously minimizing investment costs, groundwater extraction and carbon dioxide $\left(\mathrm{CO}_{2}\right)$ emissions. These objectives are selected as the focus for the analysis due to the anticipated challenges in balancing future socioeconomic development with aspirations surrounding global climate stewardship and national food security. The former is a concern due to increasingly stringent global climate change policy, and the fact that more than half of the current power generation fleet in Saudi Arabia burns extremely carbon-intensive crude oil [51]. Fulfilling national food security ambitions locally in Saudi Arabia's harsh desert environment requires industrial-scale irrigation, and has driven widespread over-exploitation of regional groundwater resources, leading to concerns regarding long-term supply sustainability [52]. Cost, emissions and groundwater criteria are accounted for in the SEWP model by tracking the corresponding cumulative value over the planning horizon (2010-2050) and over all sub-national regions (13 provinces).

The case study in this paper demonstrates the analytical efficiency of a multi-objective framing to long-term planning models of water and energy supply systems, and is applied within a scenario analysis involving interactive specification of the criteria aspiration and reservation levels. Relative levels of ambition across the disparate objectives are defined by normalizing the range between the nadir and utopia values for each criteria, and separating the normalized values into three intervals: Ambitious (+++), Moderate $(++)$, and Relaxed $(+)$. The Ambitious criteria interval has the aspiration and reservation levels near the utopia point, whereas the Relaxed interval converges on the nadir. Scenarios involving a combination of these aspiration and reservation categories are initially defined to explore trade-offs between sustainability objectives. Following the initial assessment, a sensitivity analysis is performed in which approximately 100 model iterations are explored (i.e., criteria preferences specified by diverse combinations of the aspiration and reservation levels).

Technology performance and demands for electricity and water occurring in the agricultural, municipal and manufacturing sectors are key inputs to the MCA framework. The analysis in this paper focuses on a single technology performance scenario; sensitivity of the SEWP model to these assumptions were explored previously [28]. Exogenous demands from each sector are generated with quantitative socioeconomic projections that follow the Shared Socioeconomic Pathways (SSP) [53]. National population and per capita GDP increase more than two-fold by 2050 in the mid-range (SSP2) scenario [54-56]. Previously derived sector-specific econometric models linking population and GDP to freshwater and electricity demand are used to convert the SSP data into provincial demand trajectories [28]. Moderate levels of end-use technological change are included, and reflect expected efficiency improvements driven by technological innovation. The SSP2 scenario results in modeled national electricity demands (other than for water supply) increasing from approximately $200 \mathrm{TWh}$ in 2010 to more than $700 \mathrm{TWh}$ in 2050 . Freshwater demands (other than for power supply) increase less dramatically, from $21 \mathrm{~km}^{3}$ in 2010 to $25 \mathrm{~km}^{3}$ in 2050 , due to anticipated impacts of existing national agricultural policy [57]. A detailed account of the input data used to parameterize the model, including an assessment of existing infrastructure, can be found in [28]. 

cost-minimization solution.

\begin{tabular}{|c|c|c|c|c|c|c|c|c|c|c|c|c|}
\hline \multirow{3}{*}{ Scenario name } & & & & \multicolumn{9}{|c|}{ Criteria reservation $(q)$, aspiration $(\bar{q})$ and outcome $(q)$} \\
\hline & \multicolumn{3}{|c|}{ Criteria ambition-level } & \multicolumn{3}{|c|}{ Cost $\left[\times 10^{12}\right.$ USD $]$} & \multicolumn{3}{|c|}{$\mathrm{CO}_{2}\left[\times 10^{9}\right.$ metric tons $]$} & \multicolumn{3}{|c|}{$\mathrm{GW}\left[\times 10^{3} \mathrm{~km}^{3}\right]$} \\
\hline & Cost & $\mathrm{CO}_{2}$ & GW & $\underline{q}$ & $\bar{q}$ & $q$ & $\underline{q}$ & $\bar{q}$ & $q$ & $\underline{q}$ & $\bar{q}$ & $q$ \\
\hline Cost selfish & $(+++)$ & $(-)$ & $(-)$ & 1.04 & 0.24 & 0.24 & - & - & 8.32 & - & - & 1.26 \\
\hline $\mathrm{CO}_{2}$ selfish & $(-)$ & $(+++)$ & $(-)$ & - & - & 1.25 & 3.51 & 0.46 & 0.46 & - & - & 0.21 \\
\hline GW selfish & $(-)$ & $(-)$ & $(+++)$ & - & - & 2.17 & - & - & 4.04 & 0.39 & 0.03 & 0.03 \\
\hline GW-CO $\mathrm{CO}_{2}$ ambitious & $(+)$ & $(+++)$ & $(+++)$ & 2.05 & 0.24 & 0.81 & 2.75 & 0.46 & 1.18 & 0.30 & 0.03 & 0.12 \\
\hline Cost- $\mathrm{CO}_{2}$ ambitious & $(+++)$ & $(+++)$ & $(+)$ & 0.84 & 0.24 & 0.53 & 2.66 & 0.46 & 1.52 & 0.84 & 0.03 & 0.42 \\
\hline Cost-GW ambitious & $(+++)$ & $(+)$ & $(+++)$ & 0.84 & 0.24 & 0.56 & 7.31 & 0.46 & 4.07 & 0.30 & 0.03 & 0.17 \\
\hline $\mathrm{CO}_{2}$ ambitious & $(++)$ & $(+++)$ & $(++)$ & 2.05 & 0.24 & 0.69 & 2.75 & 0.46 & 1.04 & 0.84 & 0.03 & 0.23 \\
\hline GW ambitious & $(++)$ & $(++)$ & $(+++)$ & 2.05 & 0.24 & 0.74 & 7.31 & 0.46 & 2.37 & 0.30 & 0.03 & 0.11 \\
\hline Cost ambitious & $(+++)$ & $(++)$ & $(++)$ & 0.84 & 0.24 & 0.47 & 7.31 & 0.46 & 3.04 & 0.95 & 0.03 & 0.38 \\
\hline Cost-GW-CO ${ }_{2}$ & $(+++)$ & $(+)$ & $(++)$ & 0.84 & 0.24 & 0.50 & 7.31 & 0.46 & 3.40 & 0.64 & 0.03 & 0.29 \\
\hline Cost- $\mathrm{CO}_{2}-\mathrm{GW}$ & $(+++)$ & $(++)$ & $(+)$ & 0.84 & 0.24 & 0.49 & 5.03 & 0.46 & 2.32 & 0.95 & 0.03 & 0.41 \\
\hline All criteria ambitious & $(+++)$ & $(+++)$ & $(+++)$ & 0.48 & 0.24 & 0.62 & 1.38 & 0.46 & 1.89 & 0.15 & 0.03 & 0.22 \\
\hline
\end{tabular}

Table 1: Parameterization of the decision-making preferences (aspiration and reservation levels) and the corresponding MCA results for the preliminary scenarios investigated. Each scenario is identified based on its level of ambition with respect to cost, $\mathrm{CO}_{2}$ and groundwater ( $\mathrm{GW}$ ) objectives. Relative levels of ambition across the disparate objectives are defined by normalizing the range between the nadir and utopia values for each criteria, and separating the normalized values into three intervals: Ambitious $(+++)$, Moderate $(++)$, and Relaxed $(+)$; inactive criteria are marked by (-). The Ambitious criteria interval has the aspiration and reservation levels near the utopia values, whereas the Relaxed interval converges on the nadir.

\section{Results}

This section presents key results of the scenario analysis with specific focus on the impacts of the MCA enhancements on system cost in the SEWP model. To highlight system boundaries, the scenario analysis initially involves exploration of the utopia solutions, and then moves to adjusting the aspiration and reservation levels to explore compromise solutions. Outcomes for each criteria for a select range of aspiration and reservation levels obtained through interactive scenario analysis are presented in Table 1. The relationship between the criteria for the selected scenarios are also plotted in Figure 1, where results are indexed to the respective criteria outcome obtained in the (1)

We find largest cost trade-offs in this preliminary analysis for the groundwater selfish scenario. Under the parameterized technology costs, this scenario represents a discounted system cost that is more than 8 times the costminimization (cost selfish) solution. In fact, the cost selfish solution corresponds to the groundwater nadir outcome, highlighting the direct trade-offs between these objectives. The $\mathrm{CO}_{2}$ selfish solution is also more than 6 times expensive than the cost-minimization solution; however, this scenario also achieves groundwater co-benefits, as indicated by the $80 \%$ drop in cumulative groundwater extraction compared to the cost-minimization solution (Figure 1). Varying the criteria aspiration and reservation levels across the other scenarios listed in Table 1 reveals that the largest costs are incurred when fulfilling the stringent $\mathrm{CO}_{2}$ and groundwater preferences, and that a slightly relaxed criteria preference can achieve significant cost savings while remaining ambitious from an environmental perspective. For example, when all criteria are set to relatively ambitious preferences (i.e., the 'all criteria ambitious' scenario), the MCA model 


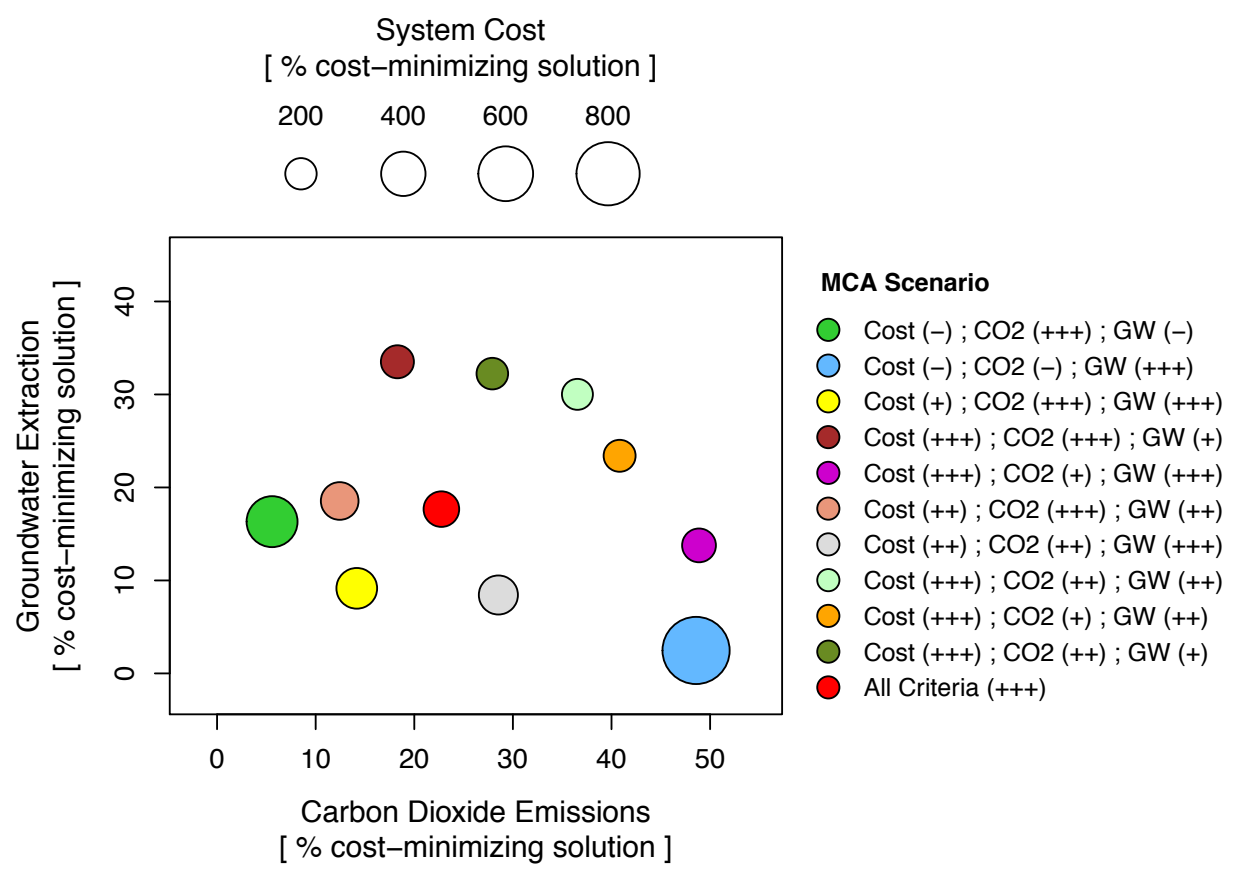

Figure 1: Investment cost, groundwater extraction and $\mathrm{CO}_{2}$ emission outcomes obtained for the scenarios listed in Table 1. The marker area is proportional to the discounted system cost. Results are indexed to the respective criteria outcome obtained in the cost-minimization solution.

seeks a Pareto-optimal solution that is relatively balanced across objectives. Pareto-optimal in this context refers to a solution where there exists no other solution for which one of the criterion (i.e., discounted costs, $\mathrm{CO}_{2}$ emissions and groundwater extraction) can be made better without sacrificing performance of the other criteria. The discounted system cost in this solution is only 2.5 times the cost-minimization outcome, but simultaneously achieves deep reductions in cumulative groundwater extraction (more than $80 \%$ reduction versus the cost-minimization outcome) and cumulative $\mathrm{CO}_{2}$ emissions (more than $75 \%$ reduction versus the cost-minimization outcome). Further relaxing the cost preferences (i.e., the 'GW- $\mathrm{CO}_{2}$ ambitious' scenario) results in a system that is 3.4 times more expensive than the cost-minimization solution, but achieves a further $10 \%$ reduction in cumulative groundwater extraction and $\mathrm{CO}_{2}$ emissions. The level of mitigation in this latter scenario is likely required to avoid local groundwater shortages [58], and achieve national electricity sector contributions to global climate stabilization [59].

\subsection{Impact of criteria preferences on system configuration}

Impacts of the criteria settings on the provincial-level technology build-out for selected scenarios are provided in Figure 2. Depicted is the optimal annual electricity and freshwater supply mix in each region, as well as the interprovincial transfers and demand-levels. The cost-minimization solution (Figure 2a) involves expansion of relatively low-cost combined-cycle natural gas generation, with existing renewable energy policy driving development of 50 GW of mostly solar generation capacity. Groundwater withdrawals are left unconstrained in the cost-minimization 
model, and under the parameterized costs dominate the future water supply mix and displace existing interprovincial desalination transfers. Moreover, in the cost-minimization solution thermal power plants employ once-through freshwater cooling systems due to the low investment cost and lack of concern surrounding groundwater sustainability. The modeled extraction across sectors in this scenario likely exceeds available aquifer storage [58].

In the groundwater selfish solution (Figure $2 b$ ) costs are more than 8 times the cost-minimization solution due to the rapid expansion of desalination, wastewater recycling and rainwater harvesting, and corresponding development of highly integrated interprovincial conveyance networks to meet water demands located inland. The increased electricity load from the water sector technologies increases aggregate national electricity demand in 2050 by $12 \%$ compared to the cost-minimization solution, and additional electricity sector capacity is developed to meet these requirements. Deep reductions in technology costs projected later in the simulation horizon combined with a lack of water requirements results in solar PV dominating the 2050 electricity supply mix in the groundwater selfish solution, and large-scale investment into electricity storage and load control capacity enables this transition (not depicted).

Similar characteristics of the 2050 supply mix are apparent when all criteria are set to ambitious preferences (Figure 2c). The push to reduce costs in this scenario results in a slower transition away from groundwater extraction and $\mathrm{CO}_{2}$ emissions, and enables groundwater and fossil fuel generation to continue to provide services in areas facing costly infrastructure constraints. For example, inland provinces continue to extract groundwater in the 'all criteria ambitious' scenario to displace investment in rainwater harvesting and conveyance infrastructure, and fossil fueled power plants are operated to provide flexibility to displace investment in storage technology and transmission upgrades.

\subsection{Sensitivity analysis}

The sensitivity analysis involved over 100 model iterations (i.e., preferences specified by diverse combinations of the aspiration and reservation levels). Each of the identified Pareto-optimal solutions has a certain trade-off (compromise) between criteria values. However, in decision-making practice extreme solutions (i.e., solutions with very good values for some criteria and very bad for the other criteria) are rarely accepted. As an example of exploration of criteria trade-offs we examine the iterations presented in Figure 3. The solutions are sorted by increasing cost.

Similar to the preliminary analysis, solutions with low cost have very high levels of $\mathrm{CO}_{2}$ emissions and groundwater extraction. For a relatively small increase of cost one can achieve substantial reduction to the other two criteria, although such reductions are not monotone for both criteria. On the other hand, solutions with very low levels of $\mathrm{CO}_{2}$ and groundwater are very expensive. This illustration of various efficient solutions provides a good basis for selecting a subset of the Pareto-frontier for further exploration. Such a selection depends on the preferences of actual decision-makers.

In a real-world planning scenario, the results of the sensitivity analysis can be presented to decision-makers who decide on the actual available budget and the goals for the other criteria. The primary role of the MCA is to help these decision-makers identify goals for all criteria that are simultaneously attainable. The MCA scenarios aligned 

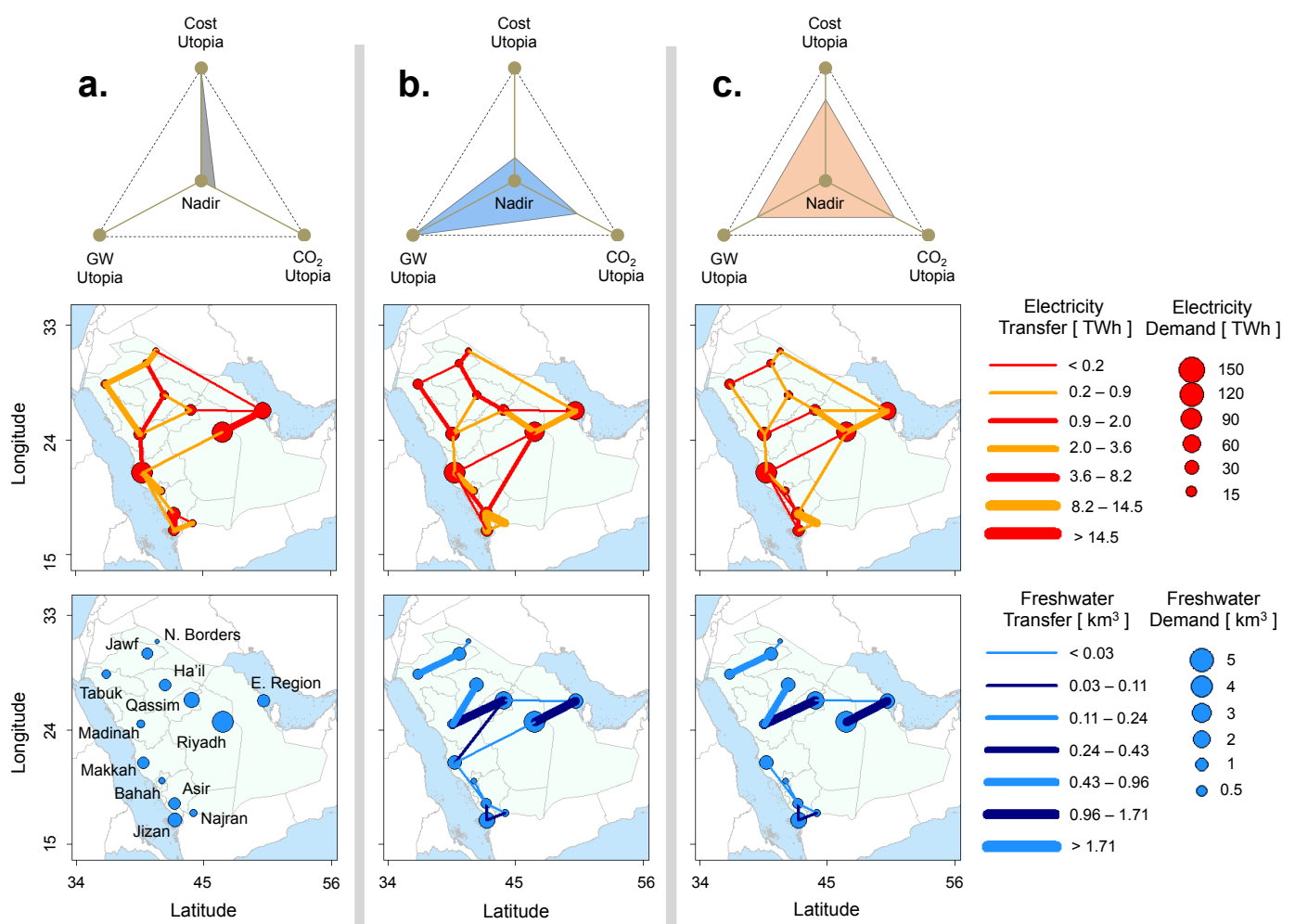
Transfer [ TWh ] Demand [ TWh ]
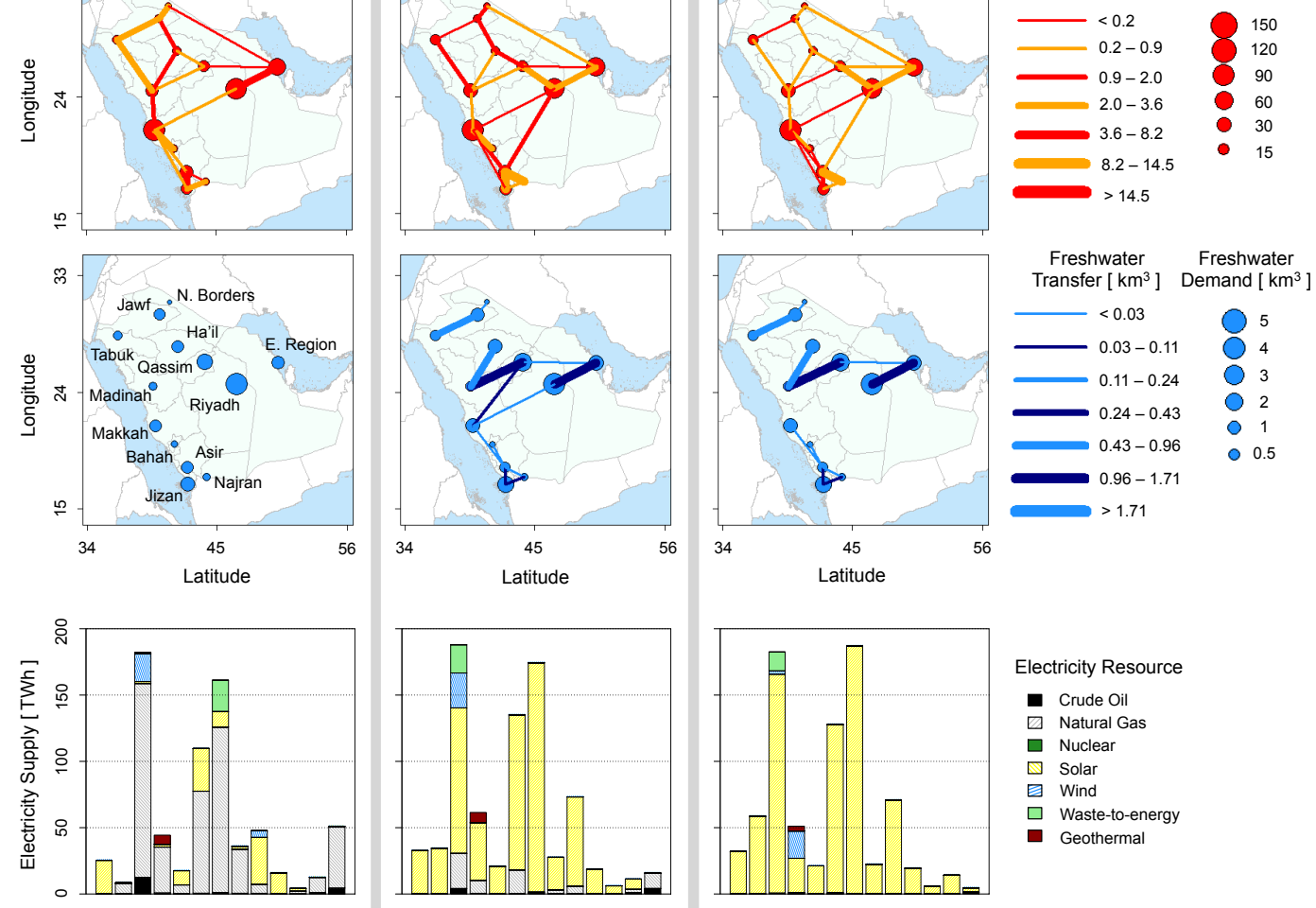

Electricity Resource

- Crude Oil

Datural Gas

$\square$ Nuclear

$\square$ Solar

Wind

$\square$ Waste-to-energy

Geothermal
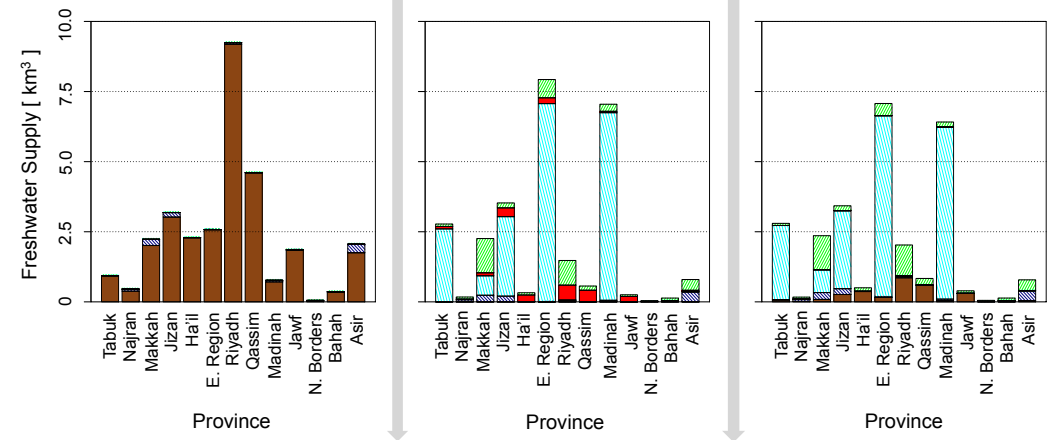

Freshwater Resource

Groundwater

Surface Water

$\boxminus$ Desalination

- Rainwater Harvesting

Wastewater Recycling

Figure 2: Provincial electricity and freshwater supply in 2050 for three of the MCA scenarios listed in Table 1. a. Cost selfish (minimization) solution; b. Groundwater (GW) selfish solution; c. All criteria ambitious solution. The top row depicts the criteria outcomes in relation to the Utopia and Nadir points. Row two and three from the top depict the annual freshwater and electricity transfers between provinces, as well as the scale of annual demand. The bottom two rows depict the supply mix from the different resources. 

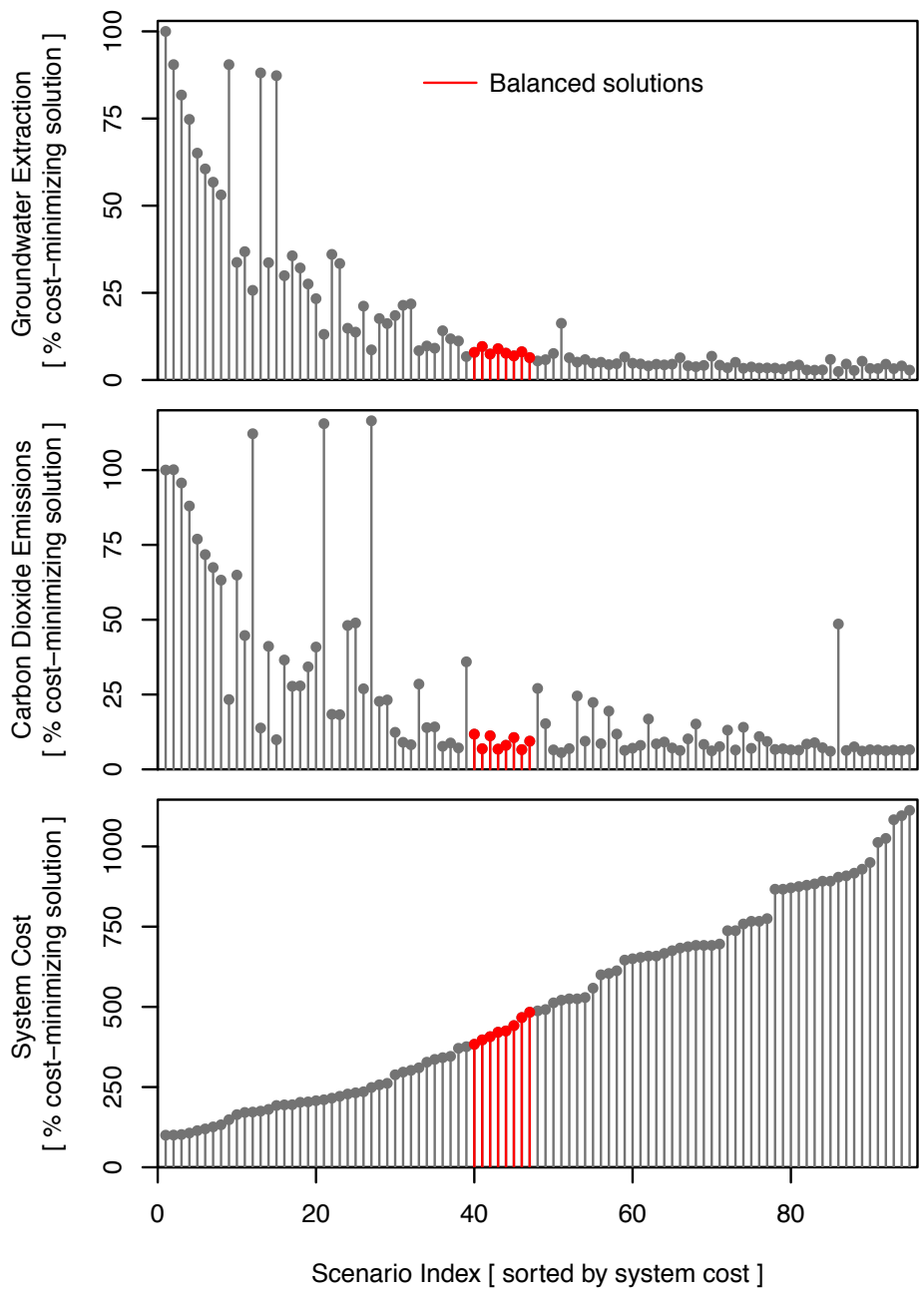

Figure 3: Criteria outcomes for the extended scenario analysis and identification of potential balanced solutions. Results are indexed to the respective criteria outcome obtained in the cost-minimization solution. 
with the decision-makers' preferences would then be further vetted with detailed operational analysis and stakeholder involvement [60].

For example, solutions in the region marked as balanced solutions in Figure 3 might be considered as having good compromises between the criteria values, as each of them achieves relatively ambitious outcomes for both groundwater and $\mathrm{CO}_{2}$ with relatively moderate impact on costs. Mitigation costs increase rapidly for more expensive solutions with relatively little improvement over the other criteria, and can therefore be deemed cost-prohibitive. Balanced solutions display similar system configurations in 2050 as in (Figure 2c), but are distinct with respect to implementation time. Largest cost savings are found to accompany balanced solutions that wait longest to transition away from groundwater.

\section{Conclusion}

Water and energy systems are increasingly interdependent, and will benefit from integrated long-term development strategy. Diverse performance criteria across development objectives necessitate multi-criteria assessment methods and tools. This paper presented a multi-criteria model analysis framework for long-term energy and water supply planning at national or regional scales. The framework incorporates a linear systems-engineering model of the coupled supply technologies and intra-regional transmission networks. A modified version of the reference point methodology enables interactive specification of decision-making preferences for disparate sustainability criteria, and convergence on a Pareto-optimal solution reflecting the relative criteria ambition-levels. Scenarios involving a combination of economic, climate and groundwater sustainability preferences were explored in the context of national planning in Saudi Arabia to demonstrate the performance of the novel analysis framework, as well as to quantify criteria tradeoffs specific to the case study region.

Application of the integrated modeling framework in the case study region demonstrates important tradeoffs between diverse sustainability criteria. Similar to previous research [28], we find that policy objectives in Saudi Arabia for 2050 that reduce cumulative groundwater extraction and electricity sector $\mathrm{CO}_{2}$ emissions to levels likely needed to avoid local groundwater shortages and meet global climate stabilization targets are associated with a significant increase in system investment costs. However, the MCA framework in this paper goes further by revealing a suite of trade-off solutions that remain nearly ambitious at much lower costs. These savings would impact the affordability of water and energy services in the rapidly developing nation of Saudi Arabia. This result is relevant from a policy-perspective because it underscores the importance of identifying a suitable compromise between sustainability objectives during the formulation of long-term water and energy strategy.

Our results further demonstrate that a conventional linear systems-engineering model used to identify optimal capacity expansion policies and investment strategies for integrated water-energy systems can be efficiently converted into a multi-objective framework using a generic transformation algorithm. Overall, the MCA framing is found to require approximately the same computational effort to solve each scenario as the single-objective framing, with the added benefits of significant analytical efficiency in terms of long-term performance assessment due to the capabilities 
in balancing multiple development objectives. It is therefore recommended that regional policy-makers incorporate similar MCA methods into their assessment of long-term water and energy strategy.

The scope of model applications in this paper focuses mainly on the electricity sector. Future work should consider expanding the system boundaries to allow assessment from resource extraction through to end-use services. This would allow mapping the impacts from a more comprehensive set of technologies and demand management policies to energy and water sustainability metrics of interest. An important issue to address in this context is the linking of surface and groundwater management, which was simplified in the analysis due to surface water scarcity in the case study region. Moreover, the effects of other criteria important to regional planners (e.g., air pollution, energy security, investment risk, climate change impacts, etc.) on the optimal development strategy should be explored to fully highlight potential trade-offs or synergies. The general MCA framework proposed in this paper can readily be adapted to include these features, and will be the topic of future research.

\section{Appendix A. MCA process and implementation}

This supplementary material describes in greater detail the MCA procedure applied in this paper and its implementation as an integrated software tool. This framework is embedded in the modular web-based tool for multiple criteria model analysis (MCMA) [50].

\section{Appendix A.1. Process}

Specification of the MCA starts with uploading the core model provided either in the standard mathematical programming system (MPS) format or as a General Algebraic Modeling System (GAMS) format model. In this paper, the core model is written in the GNU mathematical prgramming language and converted to MPS format. The names of the core model variables are presented to the user, who selects those to be used as criteria, and defines the corresponding criterion name and type (either minimization or maximization). The uploaded core model together with the criteria specification constitutes the MCA problem instance, definition of which triggers a set of optimization tasks necessary for computing the pay-off table, i.e., the values of utopia components and an approximation of the nadir Computation of the pay-off table requires $4 \cdot K$ optimizations, where $K$ is the number of selected criteria. After these computations are completed, the MCA problem instance is ready for interactive analysis. An option for defining more than one analysis instance is used in diverse situations, e.g., when problems are analyzed by several users or if a user wants to make several analyses each with a different focus. The initial analysis instance is generated automatically. Subsequent instances are optionally created by the users whenever desired.

MCA is an iterative process supporting the user in the Pareto set exploration that aims at finding subsets of solutions with desired properties (e.g., cheap, or moderately priced, or expensive). Therefore each analysis is composed of iterations. To provide an initial view on the Pareto-set, several iterations are generated automatically. First, efficient 
solutions corresponding to each utopia component are generated by selfish optimization of the corresponding criterion, i.e., all other criteria are set to be inactive. Finally, an example of balanced preferences is generated by setting for each criterion the same relative (to the utopia/nadir range) levels of aspiration and reservation.

With the above summarized background information the user takes full control of further iterations. For each iteration the user analyzes the Pareto-solutions obtained in previous iterations, and considers which criteria he/she wants to improve and which should be compromised, and then sets values for each criterion of aspiration and reservation aiming at obtaining an efficient solution that fits their preferences (desired trade-offs between criteria values) better. At each iteration the multi-criteria problem is converted into an auxiliary parametric single-objective problem using the achievement scalarizing function given by (10), the solution of which provides a Pareto solution hopefully having a better trade-off between criteria than the previous solution.

Typically, the MCA users explore various areas of the Pareto frontier (e.g., cheap and expensive having the corresponding bad and good values of environmental criteria) before deciding which compromises between the criteria values fit best their preferences. Examples of this process are provided in Section 4, and more methodological background in $[32,37,48,49]$

\section{Appendix A.2. Implementation}

The MCA of the model described in Section 2.2 was done with the MCMA, modular web-based tool for multiple criteria model analysis [50]. The MCMA tool implements the methodology described in Section 2.2 and enables analysis of models provided in either the standard MPS format for linear programming (LP) models or models specified in GAMS. In order to enable a proper MCA the core models should conform to specific requirements on the core model (i.e., outcome variables defined, no constraints due to preferences, optimization criterion ignored, etc.).

The workflow of the MCA implementation is actually hidden from the MCA users, who are guided through the MCA process (described in Appendix A.1) by a typical Graphical User Interface (GUI). The SEWP core model described in Section 3 is initially generated in the standard MPS format in the same way as for the traditional singlecriterion optimization; only the constraints for objectives other than cost are not generated. Then the MCMA tool is used for the MCA process described in Appendix A.1. For each iteration (i.e., specification of aspiration and reservation values for each criterion) the following actions are executed:

- The interactively specified values of $\bar{q}_{k}$ and $\underline{q}_{k}$ are stored in a common data-base (DB).

- The GUI calls the multi-criteria (MC)-solver, which generates the MC-part of the MCA, and queues the corresponding Optimization Task (OT).

- A dedicated utility called Task Manager (TM) distributes the OTs over the workstations with the available optimizers (same solvers as used for the single criterion model optimization). 
- A dedicated MC optimization-solver merges the MC-part with the core model into either the MPS standard file or a GAMS format model, and invokes the relevant solver for solving the corresponding LP problem. For the MCA of the SEWP model, the CPLEX solver is used.

- After the LP problem is solved, the MCO-solver extracts from the provided solution file values of criteria and uploads them into the DB.

- After the solution is uploaded into the DB, the MC-solver computes the elements of the graphical solution representation, and marks in the DB as available for the user.

- The status of computations related to each MCA iteration is updated in the DB by each software component. The GUI checks this status whenever the user wants to explore the results of the corresponding iteration, and provides the user with access to the relevant selected iteration of efficient solutions or to the information about the computation status of the iteration.

- In addition to the analysis in the criteria space typically supported by the GUI of the MCA tools, the user has access to full solutions provided by the solver of the optimization task. These solution can therefore be used for model-specific analysis (a sample of such analysis is shown in Section 4).

\section{Acknowledgements}

SCP was supported in part by a post-graduate scholarship from the Natural Sciences \& Engineering Research Council of Canada. ND acknowledges with thanks funding of this project by the Deanship of Scientific Research (DSR), King Abdulaziz University, Jeddah, under grant no. (1-135-36-HiCi).

\section{References}

[1] IEA, Chapter 17: Water for Energy, in: World Energy Outlook, International Energy Agency, 2012, pp. 1-33.

[2] S. G. Rothausen, D. Conway, Greenhouse-gas emissions from energy use in the water sector, Nature Climate Change 1 (4) (2011) $210-219$.

[3] A. J. Seebregts, G. A. Goldstein, K. Smekens, Energy/environmental modeling with the MARKAL family of models, in: Operations Research Proceedings 2001, Springer, 2002, pp. 75-82.

[4] L. Schrattenholzer, A. Miketa, K. Riahi, R. A. Roehrl, Achieving a sustainable global energy system: Identifying possibilities using long-term energy scenarios, Edward Elgar Publishing, 2004.

[5] M. Howells, H. Rogner, N. Strachan, C. Heaps, H. Huntington, S. Kypreos, A. Hughes, S. Silveira, J. DeCarolis, M. Bazillian, et al., OSeMOSYS: The open source energy modeling system: An introduction to its ethos, structure and development, Energy Policy 39 (10) (2011) 5850-5870.

[6] S. Atilhan, A. B. Mahfouz, B. Batchelor, P. Linke, A. Abdel-Wahab, F. Nápoles-Rivera, A. Jiménez-Gutiérrez, M. M. El-Halwagi, A systemsintegration approach to the optimization of macroscopic water desalination and distribution networks: a general framework applied to Qatar's water resources, Clean Technologies and Environmental Policy 14 (2) (2012) 161-171.

[7] M. Fripp, Switch: a planning tool for power systems with large shares of intermittent renewable energy, Environmental science \& technology 46 (11) (2012) 6371-6378. 
[8] E. H. Beh, G. C. Dandy, H. R. Maier, F. L. Paton, Optimal sequencing of water supply options at the regional scale incorporating alternative water supply sources and multiple objectives, Environmental Modelling \& Software 53 (2014) 137-153.

[9] M. T. van Vliet, S. Vögele, D. Rübbelke, Water constraints on European power supply under climate change: Impacts on electricity prices, Environmental Research Letters 8 (3) (2013) 035010.

[10] S. J. Pereira-Cardenal, H. Madsen, K. Arnbjerg-Nielsen, N. Riegels, R. Jensen, B. Mo, I. Wangensteen, P. Bauer-Gottwein, Assessing climate change impacts on the Iberian power system using a coupled water-power model, Climatic Change (2014) 1-14.

[11] A. Santhosh, A. M. Farid, K. Youcef-Toumi, Real-time economic dispatch for the supply side of the energy-water nexus, Applied Energy 122 (2014) 42-52.

[12] M. Bekchanov, C. Ringler, A. Bhaduri, M. Jeuland, How would the Rogun Dam affect water and energy scarcity in Central Asia?, Water International 40 (5-6) (2015) 856-876.

[13] S. J. Pereira-Cardenal, B. Mo, A. Gjelsvik, N. D. Riegels, K. Arnbjerg-Nielsen, P. Bauer-Gottwein, Joint optimization of regional water-power systems, Advances in Water Resources 92 (2016) 200-207.

[14] N. Buras, Integration of Water Resource Constraints in Energy Models, Tech. rep., Systems Optimization Laboratory, Department of Operations Research, Stanford University (1979).

[15] A. F. P. de Lucena, R. Schaeffer, A. S. Szklo, Least-cost adaptation options for global climate change impacts on the Brazilian electric power system, Global Environmental Change 20 (2) (2010) 342-350.

[16] M. Webster, P. Donohoo, B. Palmintier, Water-CO2 trade-offs in electricity generation planning, Nature Climate Change 3 (12) (2013) 10291032 .

[17] S. Bouckaert, E. Assoumou, S. Selosse, N. Maïzi, A prospective analysis of waste heat management at power plants and water conservation issues using a global TIMES model, Energy 68 (2014) 80-91.

[18] S. M. Cohen, K. Averyt, J. Macknick, J. Meldrum, Modeling climate-water impacts on electricity sector capacity expansion, in: ASME 2014 Power Conference, American Society of Mechanical Engineers, 2014, pp. 1-8.

[19] N. Johnson, O. Fricko, S. Parkinson, K. Riahi, Energy sector adaptation in response to water scarcity, in: AGU Fall Meeting, American Geophysical Union, 2015.

[20] S. C. Parkinson, N. Djilali, Robust response to hydro-climatic change in electricity generation planning, Climatic Change 130 (4) (2015) 475-489.

[21] Z. Khan, P. Linares, J. García-González, Adaptation to climate-induced regional water constraints in the Spanish energy sector: An integrated assessment, Energy Policy 97 (2016) 123-135.

[22] J. Matsumoto, L. W. Mays, Capacity expansion model for large-scale water-energy systems, Water Resources Research 19 (3) (1983) 593607.

[23] A. Dubreuil, E. Assoumou, S. Bouckaert, S. Selosse, N. Marzi, Water modeling in an energy optimization framework: The water-scarce Middle East context, Applied Energy 101 (2013) 268-279.

[24] M. D. Bartos, M. V. Chester, The conservation nexus: Valuing interdependent water and energy savings in Arizona, Environmental Science \& Technology 48 (4) (2014) 2139-2149.

[25] M. Welsch, S. Hermann, M. Howells, H. H. Rogner, C. Young, I. Ramma, M. Bazilian, G. Fischer, T. Alfstad, D. Gielen, Adding value with CLEWS: Modelling the energy system and its interdependencies for Mauritius, Applied Energy 113 (2014) 1434-1445.

[26] N. Al-Qattan, M. Ross, A. K. Sunol, A multi-period mixed integer linear programming model for water and energy supply planning in Kuwait, Clean Technologies and Environmental Policy 17 (2) (2015) 485-499.

[27] W. Huang, D. Ma, W. Chen, Connecting water and energy: Assessing the impacts of carbon and water constraints on China's power sector, In Press, Applied Energy (2016) 1-8.

[28] S. C. Parkinson, N. Djilali, V. Krey, O. Fricko, N. Johnson, Z. Khan, K. Sedraoui, A. H. Almasoud, Impacts of groundwater constraints on Saudi Arabia's low-carbon electricity supply strategy, Environmental Science \& Technology 50 (4) (2016) 1653-1662.

[29] Y. Saif, A. Almansoori, A capacity expansion planning model for integrated water desalination and power supply chain problem, Energy 
Conversion and Management 122 (2016) 462-476.

[30] D. L. McCollum, V. Krey, K. Riahi, An integrated approach to energy sustainability, Nature Climate Change 1 (9) (2011) 428-429.

[31] D. L. McCollum, V. Krey, K. Riahi, P. Kolp, A. Grubler, M. Makowski, N. Nakicenovic, Climate policies can help resolve energy security and air pollution challenges, Climatic Change 119 (2) (2013) 479-494.

[32] M. Makowski, Management of attainable tradeoffs between conflicting goals, Journal of Computers 4 (10) (2009) 1033-1042.

[33] L. Duckstein, S. Opricovic, Multiobjective optimization in river basin development, Water Resources Research 16 (1) (1980) 14-20.

[34] M. Makowski, L. Somlyódy, D. Watkins, Multiple criteria analysis for water quality management in the Nitra basin, Journal of the American Water Resources Association 32 (5) (1996) 937-951.

[35] S. Pohekar, M. Ramachandran, Application of multi-criteria decision making to sustainable energy planning: A review, Renewable \& Sustainable Energy Reviews 8 (4) (2004) 365-381.

[36] M. Lehtveer, M. Makowski, F. Hedenus, D. McCollum, M. Strubegger, Multi-criteria analysis of nuclear power in the global energy system: Assessing trade-offs between simultaneously attainable economic, environmental and social goals, Energy Strategy Reviews 8 (2015) 45-55.

[37] A. Wierzbicki, M. Makowski, J. Wessels, et al., Model-based decision support methodology with environmental applications, Kluwer Academic Publishing, 2000.

[38] D. Jornada, V. J. Leon, Robustness methodology to aid multiobjective decision making in the electricity generation capacity expansion problem to minimize cost and water withdrawal, Applied Energy 162 (2016) 1089-1108.

[39] Y. Zhou, R. S. Tol, Evaluating the costs of desalination and water transport, Water Resources Research 41 (3).

[40] B. R. Phillips, R. S. Middleton, SimWIND: A geospatial infrastructure model for optimizing wind power generation and transmission, Energy Policy 43 (2012) 291-302.

[41] A. S. Stillwell, M. E. Webber, Geographic, technologic, and economic analysis of using reclaimed water for thermoelectric power plant cooling, Environmental Science \& Technology 48 (8) (2014) 4588-4595.

[42] V. C. Tidwell, J. Macknick, K. Zemlick, J. Sanchez, T. Woldeyesus, Transitioning to zero freshwater withdrawal in the US for thermoelectric generation, Applied Energy 131 (2014) 508-516.

[43] E. Ibanez, T. Magee, M. Clement, G. Brinkman, M. Milligan, E. Zagona, Enhancing hydropower modeling in variable generation integration studies, Energy 74 (2014) 518-528.

[44] S. Sattler, J. Macknick, D. Yates, F. Flores-Lopez, A. Lopez, J. Rogers, Linking electricity and water models to assess electricity choices at water-relevant scales, Environmental Research Letters 7 (4) (2012) 045804.

[45] S. M. Lele, Improved algorithms for reservoir capacity calculation incorporating storage-dependent losses and reliability norm, Water Resources Research 23 (10) (1987) 1819-1823.

[46] N. Johnson, M. Strubegger, M. McPherson, S. C. Parkinson, V. Krey, P. Sullivan, A reduced-form approach for representing the impacts of wind and solar PV deployment on the structure and operation of the electricity system, Energy Economics In Press (2016) 1-11.

[47] A. P. Wierzbicki, The use of reference objectives in multiobjective optimization, in: Multiple Criteria Decision Making Theory and Application, Springer, 1980, pp. 468-486.

[48] J. Granat, M. Makowski, Interactive specification and analysis of aspiration-based preferences, European Journal of Operational Research 122 (2) (2000) 469-485.

[49] M. Makowski, A. P. Wierzbicki, Modeling knowledge: Model-based decision support and soft computations, in: Applied Decision Support with Soft Computing, Springer, 2003, pp. 3-60.

[50] M. Makowski, H. Ren, IIASA Integrated Modeling Environment Project - Multiple Criteria Model Analysis (MCMA), www.iiasa.ac.at/ marek/mca_doc/mcma.html, last accessed: 2016-06-12 (2016).

[51] IEA, International Energy Agency Statistics: Saudi Arabia, www.iea.org/countries/non-membercountries/saudiarabia/, last accessed: 201506-10 (2014).

[52] T. Gleeson, Y. Wada, M. F. Bierkens, L. P. van Beek, Water balance of global aquifers revealed by groundwater footprint, Nature 488 (7410) (2012) 197-200 
[53] B. C. ONeill, E. Kriegler, K. L. Ebi, E. Kemp-Benedict, K. Riahi, D. S. Rothman, B. J. van Ruijven, D. P. van Vuuren, J. Birkmann, K. Kok, et al., The roads ahead: Narratives for shared socioeconomic pathways describing world futures in the 21st century, Global Environmental Change.

[54] K. Samir, W. Lutz, The human core of the shared socioeconomic pathways: Population scenarios by age, sex and level of education for all countries to 2100, Global Environmental Change.

[55] R. Dellink, J. Chateau, E. Lanzi, B. Magne, Long-term economic growth projections in the Shared Socioeconomic Pathways, Global Environmental Change.

[56] L. Jiang, B. C. O’Neill, Global urbanization projections for the Shared Socioeconomic Pathways, Global Environmental Change.

[57] O. K. Ouda, Impacts of agricultural policy on irrigation water demand: A case study of Saudi Arabia, International Journal of Water Resources Development 30 (2) (2014) 282-292.

[58] S. Chowdhury, M. Al-Zahrani, Characterizing water resources and trends of sector wise water consumptions in Saudi Arabia, Journal of King Saud University-Engineering Sciences 27 (1) (2015) 68-82.

[59] K. Riahi, F. Dentener, D. Gielen, A. Grubler, J. Jewell, Z. Klimont, V. Krey, D. McCollum, S. Pachauri, S. Rao, Global Energy Assessment Chapter 17: Energy pathways for sustainable development.

[60] J. Swisher, G. Martino-Jannuzzi, R. Redlinger, Tools and Methods for Integrated Resource Planning: Improving Energy Efficiency and Protecting the Environment, Tech. rep., United Nations Environment Programme (1997). 